\title{
Development of Composition and Technologies of Dental Gel of Meloxicam
}

\author{
Marija N. Anurova ${ }^{1}$, Elena O. Bakhrushina ${ }^{1}$, Natalya B. Demina ${ }^{1}$, Alena Sergeevna Kashperko ${ }^{1 *}$, Ekaterina D. Shevchenko ${ }^{2}$, \\ Elizaveta V. Leshcheva ${ }^{1}$, Ivan I. Krasnyuk ${ }^{1}$, Alexander I. Bardakov ${ }^{1}$ \\ ${ }^{1}$ Sechenov First Moscow State University, Moscow, Russia; ${ }^{2}$ Eberhard Karls Universität Tübingen, Tübingen, Germany
}

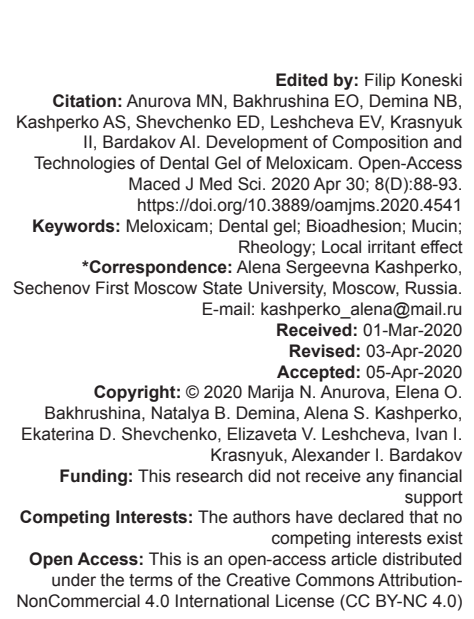

Abstract

BACKGROUND: Dental gels have several advantages over other oral dosage forms. Being a viscoplastic dosage form, the gel, when applied to the damaged area of the gum or mucous membrane, creates a protective film preventing mechanical irritation, and providing a localized effect of the drug components.

AIM: The aim of this work was to develop the composition and technology of the dental gel of meloxicam, the study of the main technological and consumer characteristics, as well as the local irritating effect of the dosage form.

METHODS: Dental gels were prepared using purified water, alcohol, glycerol, and buckthorn oil as solvents, gelling agents used were: Hydroxyethylcellulose Natrosol® $250 \mathrm{HHX}$ Pharm, Carbopol® 974P NF Polymer, and solubilizer Poloxamer 407 (Lutrol® F 127). The bioadhesive component and Noveon® Polycarbophil component were used for dental gel preparation. Aspartame was used as sweetener. Menthol and ascorbic acid were used to correct the organoleptic properties of the pharmaceutical composition. The formulated dental gel of meloxicam at a concentration of $7.5 \%$ was evaluated for organoleptic properties, $\mathrm{pH}$, rheological characteristics, bioadhesive properties, and stability under the accelerated aging period. The in vivo local irritant effect was evaluated using ten rabbits by cutaneous, subcutaneous, subconjunctival administration, as well as application to the upper palate.

RESULTS: Based on the results of studying technological and organoleptic properties, the optimal composition based on the Natrosol囚 $250 \mathrm{HHX}$ hydroxyethylcellulose gelling agent, glycerol solvent, and purified water in the ratio $1 / 5$ was selected, the composition contains Noveon ${ }^{\circledR}$ bioadhesive in an amount of $2 \%$. The composition has good taste, $\mathrm{pH}$ close to $\mathrm{pH}$ of saliva has high bioadhesive properties, satisfactory rheological characteristics. The shelf life of the experimental series by accelerated aging was 2 years. The selected composition does not have a local irritant effect.

CONCLUSION: A new dosage form of meloxicam was developed - a gel for use in dental practice.

\section{Introduction}

According to the WHO statistics, $98 \%$ of people in the world have a history of inflammatory diseases of the oral cavity, most of which are inflammatory periodontal diseases resulting in tooth loss with the absence of surgical intervention, as well as leading to morphofunctional changes in the masticatory apparatus, which are accompanied by a pain syndrome affecting the psychoemotional sphere patient activity, reduce resistance to the action of infectious and viral agents, increase the sensitization of the body, resulting in a decrease in the overall quality of life of the patient [1], [2].

In dental practice, the most common pharmacological group for relieving pain, as well as the part of complex therapy, is nonsteroidal antiinflammatory drugs (NSAIDs) [3]. The great popularity of NSAIDs is due to the fact that they have antiinflammatory, analgesic and antipyretic effects, affect the symptoms observed in a wide range of diseases. According to the FDA, the proportion of dosage forms used in dentistry based on NSAIDs is as follows: Solutions $(\sim 60 \%)$, aerosols $(\sim 25 \%)$, pastes $(\sim 8 \%)$, and gels $(\sim 7 \%)$. Thus, there is no extensive nomenclature of dental gels, although they have several advantages, specifically, they have a point effect on the site of inflammation, the absence of systemic effects due to the small mucosal coating area, as well as ease of use.

Meloxicam - carboxamide with the primary inhibitory activity of COX-2 was chosen as the object of study. In vitro studies with human tissues confirmed a high affinity of meloxicam with COX-2, while COX-1 inhibited only at the highest concentrations (ratio of $50 \%$ inhibitory concentration to COX-2: COX-1 $=0.09$ in whole blood tests). Meloxicam has a bioavailability of $89 \%$ after oral administration, is strongly associated with plasma proteins, and its half-life is $20-24 \mathrm{~h}$. It easily penetrates into the synovial fluid, reaching $45-57 \%$ of plasma concentrations [4], [5].

At present, in the development of drugs, there is a tendency to search for new potent molecules, often targeting lipophilic receptors, which in general leads to an increase in the number of highly lipophilic 
drugs with a limited therapeutic window (a low dose can be ineffective, and while a high dose leads to toxicity) [6], [7], [8], as a result of this, it was decided to use meloxicam as a weakly lipophilic drug with proven effectiveness and known toxicity.

The purpose of this study was to create a new dosage form of NSAIDs for use in dental practice, a comprehensive assessment of its physicochemical and biopharmaceutical characterization

\section{Materials and Methods}

\section{Materials}

The object of the study was the drug substance of meloxicam Boehringer Ingelheim (USP 39, Ph. Eur. 10); as solvents, the purified water (USP 39, Ph. Eur. 10), alcohol 96\% (USP 39, Ph. Eur. 10), glycerin (USP 39, Ph. Eur. 10), and buckthorn oil (SPH RF XI) were used. Other excipients used were Poloxamer 407 Lutrol@ F 127, BASF (USP 39, Ph. Eur. 10), Carbopol® 974P NF Polymer, Lubrizol (USP 39, Ph. Eur. 10), hydroxyethylcellulose Natrosol ${ }^{\circledR} 250 \mathrm{HHX}$ Pharm, Ashland (USP 39, Ph. Eur. 10), Noveon $®$ Polycarbophil, Ashland, menthol (USP 39, Ph. Eur. 10), ascorbic acid (USP 39, Ph. Eur. 10), and aspartame (USP 39, Ph. Eur. 10).

According to the published data, the concentration of NSAIDs in the developed dosage form was chosen, which amounted to $7.5 \%$ [5]. This concentration was calculated based on the average daily doses of meloxicam at the rate of $0.5 \mathrm{ml}$ of gel per dose.

First, a suspension of the active pharmaceutical ingredient was made by dispersing meloxicam in water. Gel-forming agent samples were obtained by conventional methods [9]. A gel-forming agent, flavorings, and a mucoadhesive agent were added to the resulting dispersion with continuous stirring using the ECROS ES 6120 magnetic stirrer (Russia) at a temperature of $20 \pm 2^{\circ} \mathrm{C}$. The resulting composition was left for structuring for $1.5 \mathrm{~h}$. At the end of the process, standardization was performed.

\section{Methods}

\section{Organoleptic properties}

Two methods of organoleptic evaluation were used to determine the taste of experimental samples. To determine the taste characteristics of dispersions of NSAIDs in various solvents introduced into the model gel base, the organoleptic method of taste assessment according to A.I. Tentsova. A group of twenty volunteers assesses the taste of gel samples according to the proposed scheme. The following indicators are taken into account: "Sweetness" (1 - unsweetened, and 5 very sweet), "presence of aftertaste" (1 - absent, and 5 strong present), "character of aftertaste" (1 - unpleasant, and 5 - very pleasant), and "taste in general" (1 unpleasant, and 5 - pleasant) [10]. To characterize the tastes of the experimental compositions after the initial taste correction and assess the influence of the solvent on the organoleptic properties of the finished dosage form, the profile method was used. This method is based on the construction of profilograms based on the results of assessing the taste of the compositions on a five-point scale according to indicators characterizing the taste of the compositions (acidic/bitter/sweet), the taste of the gelling agent. In addition, there is taking into account the texture indicator (numerical indices: 1 - the sign is absent; 2 - the sign is weakly expressed; 3 - a sign of normal intensity; and $4-$ a sign is very pronounced) [10].

\section{Evaluation of $\mathrm{pH}$}

When developing a dosage form for dental use, it is necessary to determine its $\mathrm{pH}$, since a low $\mathrm{pH}$ value ( $\mathrm{pH} 3-4)$ contributes to the demineralization of enamel [11]. To determine the $\mathrm{pH}$, an aqueous extraction of the gel sample under study was prepared, the resulting extraction was dispersed on an ECROS ES 6120 magnetic stirrer (Russia) for $15 \mathrm{~min}$ at room temperature, and the $\mathrm{pH}$ was determined on an Aquilon $\mathrm{pH}-410 \mathrm{pH}$ meter (Russia).

\section{Rheological properties}

A rheological study of the obtained samples was carried out on a coaxial rotational viscometer Lamy Rheology RM 200 (France) using the «cylinderin-cylinder» geometry MS-DIN 33. The results obtained were approximated with the Casson model [12], recommended for pharmaceutical compositions in the form of gels, using the Rheomatic software. To conduct the experiment, the samples are tested one by one on a viscometer at a temperature of $20^{\circ} \mathrm{C}$, which is the average temperature for storing the gel; and at a temperature of $37^{\circ} \mathrm{C}$, which is temperature of the intended application site. The plastic viscosity of the samples was studied in the range of shear rates from 0 to $300 \mathrm{~s}^{-1}$.

\section{Evaluation of bioadhesive properties}

Bioadhesion for oral dosage forms for the local use is one of the most important indicators because it determines the dose retention time on the mucous membrane, the completeness of absorption of the active substance, and the onset of the therapeutic effect.

In the experiment to determine the separation force, a lever mechanism was used, on the left shoulder of which there were movable (upper) and fixed (lower) parts, on the right - a place for cargo placement. Previously, 
non-woven material of the spanbond type was attached to the lower and upper part of the lever mechanism. A 20\% mucin solution was applied to the lower part, and a test gel sample was applied to the upper part. In this case, the mucin layer thickness was controlled, which was $0.1 \mathrm{~mm}$, which lies within the range of the mucus layer thickness under physiological conditions. A load was placed on the platform and the weight was recorded at which the upper plate was completely detached from the lower one. Bioadhesion was determined at a temperature of $20 \pm 0.5^{\circ} \mathrm{C}$, which is the storage temperature of the tested gels. The amount of adhesion was calculated as the product of the mass of sand and the acceleration of gravity $(\mathrm{g}=9.81 \mathrm{~m} / \mathrm{s} 2)$ and was expressed in newtons.

A modified model proposed by Puratchikody et al. [13] was used to determine in vitro retention times. A spanbond section (SPANLAB, Russia) with an area of $676 \mathrm{~mm}^{2}$ was attached to a glass plate measuring $260 \times 760 \mathrm{~mm}$, onto which a $4 \%$ aqueous solution of type II porcine stomach mucin was applied (SIGMA, Sigma-Aldrich, USA, Cat. No. M 2378). On a disk made of spunbond, $26 \mathrm{~mm}$ in diameter, soaked in potassium phosphate buffer solution ( $\mathrm{pH} 6.8), 1.0 \mathrm{~g}$ of the test sample was applied and uniformly distributed over the disk area. Treated spunbond membranes were combined with each other, thereby combining into a model system. Next, the resulting system was placed in the cell of the pharmacopeia apparatus of the disintegration tester of solid dosage forms ERWEKA ZT 220 "Swinging basket." The dissolution medium was potassium phosphate buffer $(\mathrm{pH} \mathrm{6.8)}$; the medium temperature was $37^{\circ} \mathrm{C}$. During the experiment, the time was noted after which the polymer disk with the test drug was torn off from the mucin-soaked spunbond.

\section{Aggregate stability}

The aggregate stability of the gel samples was assessed visually by the absence of visible changes (separation, sedimentation, and heterogeneity) and by the kinetic stability coefficient $(\mathrm{Kk})$ after centrifugation for $5 \mathrm{~min}$ at a speed of $3000 \mathrm{rpm}$ on a Biosan LMC3000 centrifuge (Germany) $5 \mathrm{ml}$ of sample gels in centrifuge tubes Greiner Bio-One $15 \mathrm{ml}$. The kinetic stability coefficient was calculated by the formula:

$$
K_{K}=\frac{\dot{I}_{1}}{\dot{I}_{2}} \text {, }
$$

where phase;

$\mathrm{H}_{1}$ - is the height of the layer of the precipitated

$\mathrm{H}_{2}$ - is the height of the gel layer.

The indicator was determined in experimental samples during storage under natural conditions (temperature $20 \pm 2^{\circ} \mathrm{C}$, and humidity 55\%).

The method of "accelerated aging" determined the shelf life of the developed dosage form. According to RSF XIV (Russia State Pharmacopoeia XIV), the limiting temperature of experimental storage was chosen $+40^{\circ} \mathrm{C}$; the thermostat Binder ED 115 (Germany) was used.

\section{Local irritant effect}

The local irritating effect was determined by the methods of cutaneous, subcutaneous, conjunctival tests, and on the oral mucosa in chinchilla rabbits weighing 3.6-4.1 kg in accordance with the "Guidelines for the Preclinical Studies of Medicines" [14]. The care and maintenance of animals were carried out in accordance with the recommendations and requirements of Directive 2010/63/EU of the European Parliament and of the EU Council of September 22, 2010 , on the protection of animals used for scientific purposes. All animal experiments were approved by the Sechenov University Ethics Committee.

Assessment of the local irritant effect on the skin was carried out on ten rabbits. Two days before the experiment, sections of $5 \times 5 \mathrm{~cm}$ in size were trimmed on the back on symmetrical sections of the back on either sides of the spine, leaving a $2 \mathrm{~cm}$ hairline between them. The right side of the animal serves to apply the studied drug, the left to control. The exposure time is $4 \mathrm{~h}$. Exposure is repeated every day for 7 days. The skin condition is recorded visually daily. Functional morphological disorders of the skin are noted in points (erythema, edema, cracks, necrosis, peeling, dryness, and ulceration). After the experiment, animals were monitored for 14 days. The test drug was added $0.5 \mathrm{ml}$ per clipped area on the right side of the rabbit. The same amount of distilled water was applied to the left side.

Subcutaneous tests were conducted on ten rabbits. Injections were administered with $0.1 \mathrm{ml}$ of gel once in shaved areas of the skin of the animal. The state of injections was noted immediately, 24, 48, and $72 \mathrm{~h}$ after administration. The degree of tissue reaction was evaluated, including erythema and edema. After the experiment, animals were also monitored for another 14 days.

The local irritant effect on the eyes was evaluated on ten rabbits. The gel in an amount of $0.1 \mathrm{ml}$ was instilled 3 times a day for 7 days. One eye is used to apply the product, the other as a control; $0.1 \mathrm{ml}$ of purified water was injected into it. The severity of hyperemia, conjunctival edema, vascular injection of the sclera, the condition of the cornea and iris, and the number and quality of discharge from the eye for 14 days, which were evaluated in points, were noted.

In addition, the local irritating effect of the gel on the mucous membrane of the oral cavity was evaluated. For this, $0.1 \mathrm{ml}$ of gel was applied to the palate of 10 rabbits 3 times a day for 7 days. To the control rabbits in the amount of ten pieces to the palate, the purified 
water was applied. The condition of the mucosa was recorded visually daily. Functional morphological disorders of the mucosa were noted in points (erythema, edema, fissures, necrosis, hyperemia, dryness, and expressions). After the experiment, animals were monitored for 14 days.

\section{Results and Discussion}

The experimental substantiation of the solvent is one of the most important steps in the development of a suitable gel formulation for local oral drug delivery. Meloxicam is practically insoluble (more than $10,000 \mathrm{~g} /$ $\mathrm{ml}$ ) in oral solvents and has a sharp taste, which may limit its use in dental practice.

Therefore, it was introduced into the following solvents as a suspension:water, ethanol, glycerol, and buckthorn oil in the range of ratios 1:5, 1:10, 1:20, 1:30, and $1: 40$. Stable for 7 days dispersions were obtained in glycerol in a ratio of 1:40 and in oil in a ratio of 1:10. To obtain a sedimentation-stable suspension in ethanol the need for the introduction of a solubilizer was identified, which was used as Poloxamer 407 Lutrol $®$ F 127 in the concentration range from $5 \%$ to $15 \%$. It was shown that a stable dispersion is formed when a solubilizer is added at a concentration of $15 \%$.

Based on the aggregate stable dispersions, experimental samples of dental gels were obtained, the composition of which is presented in Table 1. The most common and widely used gelling agents hydroxyethylcellulose Natrosol $250 \mathrm{HHX}$ (Ashland) and Carbopol@ 974P (Lubrizol) were obtained.

Dental gels must have significant bioadhesion to ensure their therapeutic effect; therefore, Noveon $\AA$ Polycarbophil (Ashland) was additionally added to formulations 5,6 , and 8 .

The next step was the determination of the organoleptic properties of the experimental gel samples, but to study the influence of solvents, solubilizer, and bioadhesive on taste, all gels were obtained on one gellant - Carbopol ${ }^{\circledR}$ 974P. To determine the taste used organoleptic method for assessing taste, according to A.I. Tentsova. The taste index was derived from the obtained results as the arithmetic mean of the obtained numerical estimates given by volunteers. A higher numerical index indicates a better masking potential of the composition. The results are shown in Table 2.

Thus, based on the taste assessment of the experimental compositions, it was found that compositions 2 and 4 require adjustments to the organoleptic properties - due to the fact that they include excipients that are not indifferent in taste and smell - the Poloxamer 407 solubilizer and ethanol as a solvent. To correct the taste, the synthetic sweetener aspartame was used at a concentration of $0.1 \%$, the acidity regulator ascorbic acid at a concentration of $0.05 \%$ and the odor flavor menthol at a concentration of $0.1 \%$ - the choice of concentrations was also carried out according to the organoleptic method A.I. Tentsova.

To characterize the tastes of the experimental compositions after conducting taste correction and assessing the effect of the gel on the organoleptic properties of the finished dosage form, the profile method was used. This method was rested on the construction of profilograms based on the results of assessing the taste of the compositions on a fivepoint scale according to indicators characterizing the taste of the compositions (acidic/bitter/sweet). In addition, the taste of the gelling agent and the indicator of texture were taking into account using numerical indices ( 1 - the sign is absent; 2 - the sign is weakly expressed; 3 - the sign of normal intensity; and 4 - sign is expressed very strongly) [10]. Profilograms of taste are presented in Figure 1.

All samples analyzed after adjusting the taste had satisfactory organoleptic characteristics. At the

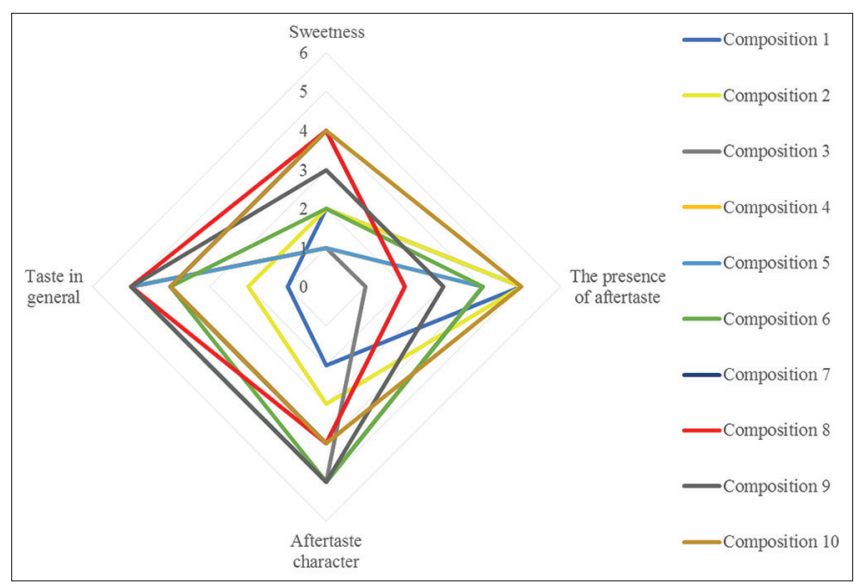

Figure 1: Profilograms of the taste of meloxicam gel samples

Table 1: The compositions of the experimental samples of meloxicam gels

\begin{tabular}{|c|c|c|c|c|c|c|c|c|c|c|}
\hline \multirow[t]{3}{*}{ Ingredients } & \multicolumn{10}{|c|}{ Number of composition } \\
\hline & \multicolumn{10}{|c|}{ Amount of ingredients, $\%$} \\
\hline & 1 & 2 & 3 & 4 & 5 & 6 & 7 & 8 & 9 & 10 \\
\hline Meloxicam & 7.50 & 7.50 & 7.50 & 7.50 & 7.50 & 7.50 & 7.50 & 7.50 & 7.50 & 7.50 \\
\hline Ethanol & 20.00 & 20.00 & - & - & - & - & - & - & - & 20.00 \\
\hline Purified water & 57.50 & 71.70 & 80.20 & 76.75 & 76.75 & 76.75 & 69.00 & 74.75 & 83.60 & 69.25 \\
\hline Poloxamer & 15.00 & - & - & - & - & - & - & - & - & - \\
\hline Carbopol & - & 0.75 & - & 0.75 & 0.75 & 0.75 & - & - & 0.75 & - \\
\hline Buckthorn oil & - & - & 7.50 & - & - & - & - & - & 8.20 & - \\
\hline Hydroxyethylcellulose & - & - & 4.50 & - & - & - & 4.00 & 4.00 & - & 3.00 \\
\hline Glycerol & - & - & - & 15.00 & 15.00 & 15.00 & 19.60 & 15.00 & - & - \\
\hline Bioadhesive & - & - & - & - & 1.00 & 2.00 & - & 2.00 & - & - \\
\hline
\end{tabular}


same time, a specific taste of the gel base was also noted in some formulations. This is more relevant for composition 4, which has a pronounced texture and a slightly bitter aftertaste. Compositions 2, 7, and 8 have a characteristic smack of a gel-forming agent of moderate intensity. According to the data obtained in the course of complex studies of the organoleptic properties of the compositions by the organoleptic method A.I. Tentsova and profile method, the most acceptable organoleptic characteristics are compounds 2,7 , and 8 .

The most important indicator in the development of gels is their aggregative stability. A measure of stability was the kinetic stability coefficient calculated as the ratio of the separated phases after centrifugation of the sample. It was accepted that the samples are stable with a kinetic stability coefficient not exceeding 0.1 (Table 3).

Compositions with buckthorn oil began to exfoliate after a week of storage. Samples using ethanol as a solvent also did not show high stability. Samples $2,4,5,7$, and 8 had satisfactory stability, since their kinetic stability coefficient did not exceed 0.1 . They also did not observe delamination, turbidity, discoloration, or gel consistency; there was no separation of the dispersion medium from the polymer network formed by the molecules of the dispersed phase (there was no syneresis).

At the next stage of the study, the physicochemical characteristics of stable meloxicam gel samples were evaluated.

According to the data obtained, it can be concluded that samples 7 and 8 have the most satisfactory $\mathrm{pH}$ values since their $\mathrm{pH}$ is as close as possible to the saliva $\mathrm{pH}(6.4-7.2)$ [15], which will not contribute to the destruction and demineralization of long-term use enamels [16]. However, the remaining samples satisfy the pharmacopeial requirements for this indicator.

Table 2: The results of determining the taste of meloxicam gel samples by A.I. Tentsova

\begin{tabular}{|c|c|c|c|c|c|c|c|c|c|c|}
\hline \multirow{2}{*}{ Indicator } & \multicolumn{10}{|c|}{ Number of composition } \\
\hline & 1 & 2 & 3 & 4 & 5 & 6 & 7 & 8 & 9 & 10 \\
\hline Sweetness & 2.0 & 2.0 & 1.0 & 1.0 & 1.0 & 2.0 & 4.0 & 4.0 & 3.0 & 4.0 \\
\hline The presence of aftertaste & 5.0 & 5.0 & 1.0 & 4.0 & 4.0 & 4.0 & 2.0 & 2.0 & 3.0 & 5.0 \\
\hline Aftertaste character & 2.0 & 3.0 & 5.0 & 5.0 & 5.0 & 5.0 & 4.0 & 4.0 & 5.0 & 4.0 \\
\hline Taste in general & 1.0 & 2.0 & 5.0 & 5.0 & 5.0 & 4.0 & 4.0 & 5.0 & 5.0 & 4.0 \\
\hline
\end{tabular}

When studying the rheological characteristics of the experimental samples, it was shown that

Table 3: Kinetic stability coefficients of meloxicam gel samples

\begin{tabular}{llllll}
\hline Sample No. & \multicolumn{5}{l}{ Shelf life, days } \\
\cline { 2 - 6 } & \multicolumn{1}{l}{ KK } & 7 & 30 & 60 & 90 \\
\hline 1 & 0.10 & 0.15 & 0.18 & 0.25 & 0.32 \\
2 & 0.00 & 0.00 & 0.00 & 0.00 & 0.09 \\
3 & 0.00 & 0.35 & 0.38 & 0.40 & 0.41 \\
4 & 0.00 & 0.00 & 0.08 & 0.09 & 0.10 \\
5 & 0.00 & 0.00 & 0.09 & 0.10 & 0.10 \\
6 & 0.00 & 0.00 & 0.12 & 0.14 & 0.26 \\
7 & 0.00 & 0.00 & 0.00 & 0.00 & 0.00 \\
8 & 0.00 & 0.00 & 0.00 & 0.00 & 0.00 \\
9 & 0.00 & 0.12 & 0.15 & 0.29 & 0.35 \\
10 & 0.00 & 0.00 & 0.08 & 0.12 & 0.28 \\
\hline
\end{tabular}

when using ethanol as a solvent of meloxicam, the yield strength characterizing the strength of the gel structure and plastic viscosity significantly differ from the performance of the samples, in which glycerol was used in various concentrations (Table 4). Thus, the introduction of ethanol in the pharmaceutical composition cannot ensure its stability during storage under shear stress (e.g., by extrusion from a tube by a patient). The rheological characteristics of the studied formulations $4,5,7$, and 8 lie in the optimum range for dental gels defined by author [12].

Then, the bioadhesive properties of meloxicam gel samples were studied using two methods: The "peel" method, where the greater the peel force, the higher bioadhesion, and the "swinging basket" method, in which the longer the retention time, the higher bioadhesion.

According to Table 5, compositions 4 and 5 based on Carbopol $\AA$ 974P have significantly lower bioadhesive retention properties than formulations 7 and 8 based on Natrosol囚 $250 \mathrm{HHX}$. The adhesion values of compositions 4 and 5 are $1.7 \pm 0.164 \mathrm{~s}$ and $2.0 \pm 0.11 \mathrm{~s}$, and for compositions 7 and 8 more than 30 times higher: $65.0 \pm 0.084 \mathrm{~s}$ and $77.0 \pm 0.071 \mathrm{~s}$, respectively. In addition, a noticeable effect on the bioadhesion of Noveon $®$ Polycarbophil administration (compositions 5 and 8). The adhesion value of composition 5 is higher by $5.6 \mathrm{~N}$ and the retention time is $0.3 \mathrm{~s}$ than that of composition 4 . For composition 8 , the adhesion value is higher by $1.2 \mathrm{~N}$ and the retention time by $12 \mathrm{~s}$. Thus, compositions 7 and 8 have the best bio-adhesive properties.

To determine the shelf life by the method of "accelerated aging," three series of samples of composition 7 and 8 were made as the most satisfactory for all previously studied indicators. For each series, the following quality indicators were evaluated during storage: Description, authenticity and quantification by HPLC, viscosity, particle size, $\mathrm{pH}$, and microbiological purity.

Table 4: Physicochemical characteristics of meloxicam gel samples

\begin{tabular}{|c|c|c|c|c|c|}
\hline \multirow{3}{*}{$\begin{array}{l}\text { Sample } \\
\text { No. }\end{array}$} & \multicolumn{5}{|l|}{ Indicator } \\
\hline & \multirow[t]{2}{*}{$\mathrm{pH} \pm \mathrm{SD}$} & \multicolumn{2}{|c|}{ Casson yield strength, $\mathrm{Pa} \pm \mathrm{SD}$} & \multicolumn{2}{|c|}{ Casson plastic viscosity, Pa.s $\pm \mathrm{SD}$} \\
\hline & & at $137^{\circ} \mathrm{C}$ & at $20^{\circ} \mathrm{C}$ & at $\mathrm{t} 37^{\circ} \mathrm{C}$ & at $20^{\circ} \mathrm{C}$ \\
\hline & 5. & 10.6 & 12 & $0.208 \pm 0.001$ & 0.001 \\
\hline Sample 4 & $5.37 \pm 0.008$ & $110.7 \pm 0.084$ & $112.5 \pm 0.084$ & $0.320 \pm 0.001$ & $0.321 \pm 0.001$ \\
\hline Sample 5 & $5.45 \pm 0.009$ & $100.5 \pm 0.071$ & $102.4 \pm 0.071$ & $0.298 \pm 0.001$ & $0.311 \pm 0.001$ \\
\hline & $6.54 \pm 0.008$ & $145.3 \pm 0.084$ & \pm 0.084 & $0.477 \pm 0.001$ & $0.487 \pm 0.001$ \\
\hline Sample 8 & $6.55 \pm 0.008$ & $148.6 \pm 0.071$ & $156.8 \pm 0.071$ & $0.485 \pm 0.001$ & $0.495 \pm 0.001$ \\
\hline
\end{tabular}

The shelf life of sample 7 was 1.5 years and the shelf life of sample 8 was 2 years, so it was considered more promising for further development. In addition, bioadhesive characteristics are also higher for composition 8.

Table 5: The results of the study of bioadhesion in samples of meloxicam gels

\begin{tabular}{lll}
\hline Sample No. & The amount of adhesion, $\mathrm{N} \pm \mathrm{SD}$ & Retention time, sec $\pm \mathrm{SD}$ \\
\hline Composition 4 & $26.095 \pm 0.004$ & $1.7 \pm 0.164$ \\
Composition 5 & $31.713 \pm 0.002$ & $2.0 \pm 0.11$ \\
Composition 7 & $29.442 \pm 0.002$ & $65.0 \pm 0.084$ \\
Composition 8 & $30.682 \pm 0.002$ & $77.0 \pm 0.071$ \\
\hline
\end{tabular}


For drugs applied to the mucous membrane, the absence of a local irritant effect is extremely important. For sample 8, the local irritant effect was evaluated in several ways.

When determining and evaluating the local irritating effect on the skin, the degree of erythema and edema was 0 , which corresponds to the absence of a local irritating effect. With intradermal administration of a dental gel with meloxicam, the degree of intradermal reactions immediately after administration is 6 points out of 8 . After $72 \mathrm{~h}$, the degree decreases to 0 , which corresponds to the absence of a local irritant effect. In the gel study to determine and evaluate the local irritant effect on the eyes, the degree of local irritant effect corresponds to "weak or absent." When this composition was applied to the mucous membrane of the oral cavity, a slight edema (0 or 1 point) or its absence, slight redness, or its absence ( 0 or 1 point) was observed, which also corresponds to the absence of a local irritant effect. Thus, a gel with meloxicam does not have a local irritant effect.

\section{Conclusions}

The meloxicam dental gel composition 8 was developed based on the Natrosol ${ }^{\circledR} 250 \mathrm{HHX}$ hydroxyethylcellulose gelling agent, glycerol solvent and purified water in a ratio of $1 / 5$ and $2 \%$ Noveon $\AA$ Polycarbophil bioadhesive was present in the composition. The composition has good taste, a $\mathrm{pH}$ close to the $\mathrm{pH}$ of saliva, high bioadhesive properties, and satisfactory rheological characteristics. The shelf life of the experimental series by accelerated aging was 2 years. The selected composition does not have a local irritant effect.

\section{References}

1. Cherkasov SM. Analysis of the prevalence of diseases of the dentofacial system, forming the demand for dental services. Fundam Res. 2014;2:186-9.

2. Miller K, Treloar T, Guelmann M, Rody W Jr., Shaddox LM. Clinical characteristics of localized aggressive periodontitis in primary dentition. J Clin Pediatr Dent. 2018;42(2):95-102. https://doi.org/10.17796/1053-4628-42.2.3 PMid:29087795

3. Monk AB, Harrison JE, Worthington HV, Teague A Pharmacological interventions for pain relief during orthodontic treatment. Cochrane Database Syst Rev. 2017;11(11):CD003976. https://doi.org/10.1002/14651858.

\section{cd003976.pub2}

PMid:29182798

4. Rigato HM, Mendes GD, Borges NC, Moreno RA. Meloxicam determination in human plasma by high-performance liquid chromatography coupled with tandem mass spectrometry (LC-MS-MS) in Brazilian bioequivalence studies. Int $\mathrm{J}$ Clin Pharmacol Ther. 2006;44(10):489-98. https://doi.org/10.5414/ cpp44489

PMid: 17063980

5. Noble S, Balfour JA. Meloxicam. Drugs. 1996;51(3):424-30 PMid:8882380

6. Hopkins AL, Keserü GM, Leeson PD, Rees DC, Reynolds CH. The role of ligand efficiency metrics in drug discovery. Nat Rev Drug Discov. 2014;13(2):105-21. https://doi.org/10.1038/ nrd4163

PMid:24481311

7. Lipinski CA, Lombardo F, Dominy BW, Feeney PJ. Experimenta and computational approaches to estimate solubility and permeability in drug discovery and development settings. Adv Drug Deliv Rev. 2001;46(1-3):3-26. https://doi.org/10.1016/ s0169-409x(96)00423-1

PMid:11259830

8. Leeson PD, Springthorpe B. The influence of drug-like concepts on decision-making in medicinal chemistry. Nat Rev Drug Discov. 2007;6(11):881-90. https://doi.org/10.1038/nrd2445 PMid:17971784

9. Anurova MN, Bakhrushina EO, Demina NB. Review of contemporary gel-forming agents in the technology of dosage forms. Pharm Chem J. 2015;49:627-34. https://doi.org/10.1007/ s11094-015-1342-5

10. Anurova MN, Bakhrushina EO, Demina NB. The problem of taste masking of drugs. Drug Dev Regist. 2015;4(13):64-73.

11. Xie WL, Chipman JG, Robertson DL, Erikson RL, Simmons DL. Expression of a mitogen-responsive gene encoding prostaglandin synthase is regulated by mRNA splicing. Proc Natl Acad Sci U S A. 1991;88(7):2692-6. https://doi.org/10.1073/ pnas.88.7.2692

PMid: 1849272

12. Anurova MN, Bakhrushina EO, Barnolitskiy GG, Krechetov SP. Justification of the rheological optimum in the development of semisolid forms. Dent Gels. 2017;2(19):58-62.

13. Puratchikody A, Prasanth VV, Mathew ST, Kumar BA. Development and characterization of mucoadhesive patches of salbutamol sulfate for unidirectional buccal drug delivery. Acta Pharm. 2011;61(2):157-70. https://doi.org/10.2478/ v10007-011-0011-9

PMid:21684844

14. Mironov AN, editors. Guidelines for Preclinical Studies of Drugs (Immunobiological Drugs). Part Two. Moscow: Grief and K; 2013.

15. Lapicque F, Vergne P, Jouzeau JI, Loeuille D, Gillet P, Vignon E, et al. Articular diffusion of meloxicam after a single oral dose: Relationship to cyclo-oxygenase inhibition in synovial cells. Clin Pharmacokinet. 2000;39(5):369-82. https://doi. org/10.2165/00003088-200039050-00005 PMid:11108435

16. Tilley SL, Coffman TM, Koller BH. Mixed messages: Modulation of inflammation and immune responses by prostaglandins and thromboxanes. J Clin Invest. 2001;108(1):15-23. https://doi. org/10.1172/jci200113416

PMid:11435451 